\title{
Extensive chaos in the Nikolaevskii model
}

\author{
Hao-wen Xi, ${ }^{1}$ Raúl Toral, ${ }^{2}$ J. D. Gunton, ${ }^{2,3}$ and Michael I. Tribelsky ${ }^{4}$ \\ ${ }^{1}$ Department of Physics and Astronomy, Bowling Green State University, Bowling Green, Ohio 43403 \\ ${ }^{2}$ Instituto Mediterráneo de Estudios Avanzados (IMEDEA), CSIC-UIB, E-07071 Palma de Mallorca, Spain \\ ${ }^{3}$ Department of Physics, Lehigh University, Bethlehem, Pennsylvania 18015* \\ ${ }^{4}$ Department of Applied Physics, Faculty of Engineering, Fukui University, Bunkyo 3-9-1, Fukui 910-8507, Japan
}

(Received 6 October 1999)

\begin{abstract}
We carry out a systematic study of a different type of chaos at onset ("soft-mode turbulence") based on numerical integration of the simplest one-dimensional model. The chaos is characterized by a smooth interplay of different spatial scales, with defect generation being unimportant. The Lyapunov exponents are calculated for several system sizes for fixed values of the control parameter $\epsilon$. The Lyapunov dimension and the Kolmogorov-Sinai entropy are calculated and both shown to exhibit extensive and microextensive scaling. The distribution functional is shown to satisfy Gaussian statistics at small wave numbers and small frequency.
\end{abstract}

PACS number(s): 05.45.- a, 47.20.Ky, 47.27.Eq, 47.52.+j

Spatiotemporal chaos (STC) is a subject of considerable experimental and theoretical importance and occurs in a wide variety of driven, dissipative systems [1-3]. Such chaotic behavior in spatially extended systems is extremely difficult to characterize quantitatively, as the dynamics involves a large number of degrees of freedom. The most common and useful tool for the characterization of chaos is given by the Lyapunov exponents $\left\{\lambda_{i}\right\}$. Knowledge of this Lyapunov spectrum permits one to estimate the number of effective degrees of freedom of the system (i.e., the dimension of the attractor), using for example the Kaplan-Yorke [4] formula for the Lyapunov dimension $D(L)$, where $L$ is the linear system size. It also permits one to test the important concept of extensivity of chaos, defined as the case in which $\lim _{L \rightarrow \infty} D(L) \sim L^{d}$, where $d$ is the spatial dimension of the system $[1,5]$. An interpretation of extensive chaos is that the whole system can then be thought of in some sense as the union of almost independent subsystems. This was originally proposed by Ruelle [6], who argued that widely separated subsystems of a turbulent system should be weakly correlated, so that the spectrum of Lyapunov exponents would be the union of exponents associated with each of the subsystems. The question is closely related to the fundamental problem of ergodicity of nonequilibrium systems. If the chaos is extensive and each subsystem evolves in time practically independently of the others, then in a steady (nontransient) chaotic state the time average is equivalent to the ensemble average and the system should be ergodic. Much work has focused on attempting to characterize spatiotemporal dynamics in these terms (see, e.g., [7-11]). However, in spite of the fundamental importance of the question practically all the results are related just to a few discrete coupled map lattices [12-14] and two continuous systems, namely, the complex Ginzburg-Landau (CGL) and KuramotoSivashinsky (KS) equations (see, e.g., Refs. $[13,15,16])$. This is partly due to the computational complexity of the problem but primarily to the lack of simple models exhibiting the

\footnotetext{
*Permanent address.
}

requisite chaotic behavior. It would therefore be of considerable interest to characterize quantitatively other types of STC.

Recently attention was drawn to the existence of a new wide class of systems displaying such a behavior [17-20]. Their properties are qualitatively different from those of the CGL and KS models. In contrast to both these models the chaos is associated with smooth, random long-wavelength modulations of a short-wavelength pattern, with defect generation being unimportant. The short-wavelength pattern arises due to a single supercritical bifurcation of the Turing type such as occurs in Rayleigh-Bénard convection. The long-wavelength modes belong to a Goldstone branch of the spectrum originated in a broken continuous symmetry. The symmetry makes the system degenerate to the extent that instead of a single, unique spatially uniform state, it has a continuous family of equivalent spatially uniform states, which may be obtained from each other by the symmetry transformation. This symmetry, which is additional to the trivial groups of translations and rotations, can be one of many different types. For this reason the STC in question is quite a common phenomenon and occurs, for example, in electroconvection in liquid crystals $[17,18]$, in convection in a fluid with stress-free boundary conditions [21-24], etc.; see Ref. [20] for further discussion. The chaos observed in such cases may be interpreted as a macroscopic dynamical analog of second-order phase transitions, where the order parameter is related to the amplitudes of turbulent modes. Due to this analogy it has been called soft-mode turbulence (SMT) [18]. The simplest model exhibiting SMT was introduced by Nikolaevskii $[25,26]$ to describe longitudinal seismic waves in viscoelastic media. In what follows we exploit the simplicity of this model to shed light on general features of this different type of STC.

We present a detailed systematic study of the Nikolaevskii model, including calculation of the Lyapunov exponents, fractal dimension, etc. We show that the Lyapunov dimension and Kolmogorov-Sinai entropy are extensive quantities, which supports the validity of the Ruelle's ideas for SMT. We also show that the power spectrum can be 
described by Gaussian statistics for small wavenumbers and frequencies, in agreement with a general argument of Hohenberg and Shraiman [5].

The model is defined by the following partial differential equation for the real scalar field $v(x, t)$ (longitudinal mode of the displacement velocity in the original formulation) $[25,26]$ :

$$
\frac{\partial v}{\partial t}+\frac{\partial^{2}}{\partial x^{2}}\left[\epsilon-\left(1+\frac{\partial^{2}}{\partial x^{2}}\right)^{2}\right] v+v \frac{\partial v}{\partial x}=0
$$

with $0 \leqslant x \leqslant L$ and periodic boundary conditions. This model has two control parameters, $\epsilon$ (the distance from onset) and $L$, in contrast to, e.g., the KS model, where the only nontrivial control parameter is the system size $L$. An essential feature of the model is that even at small $\epsilon$ it cannot be reduced to any $\epsilon$-free form [20,27], which makes the hierarchy of characteristic scales much more complex than those in the KS and CGL models [28,29]. Equation (1) may be regarded as a generalized Burgers equation and shares with it the same group of symmetry, namely, trivial symmetries under shifts of the spatiotemporal coordinate system, and nontrivial invariance with respect to the Galilean transformation $v(x, t) \rightarrow v\left(x-v_{o} t, t\right)+v_{o}$, where $v_{o}$ is an arbitrary constant. The Galilean invariance plays the role of the above specified additional symmetry, generating for Eq. (1) the continuous family of solutions $v=v_{o}$. The Nikolaevskii equation admits a continuous set of spatially periodic, stationary solutions, whose instability has been proved analytically [28]. Computer simulations [19] of an equivalent version of this model for an order parameter $u(x, t) \equiv \int v(x, t) d x$ showed that even at extremely small $\epsilon=10^{-4}$, the system exhibits STC. However, this simulation did not provide any quantitative results about the STC. The only result of this kind is in Ref. [30], in which just a single quantitative characteristic, namely, the dependence of the mean amplitude of chaotic patterns on the control parameter, was studied. In this Rapid Communication we span the gap in our knowledge of this type of STC, providing a detailed quantitative description of its most important properties based on numerical integration of Eq. (1). The simulations were carried out using the pseudospectral method combined with a fourth-order predictor-corrector integrator, for several different values of $L$ and two values of $\epsilon$ (0.2 and 0.5 , respectively) [31]. The Lyapunov exponents were calculated by linearizing the equation along the trajectory, performing a reorthonormalization after a few integration steps to prevent the largest Lyapunov exponent from swamping all the others [32]. A typical pattern $v(x, t)$ as a function of space and time in the steady chaotic regime is shown in Fig. 1. The time averaged power spectrum $\left\langle|\hat{v}(k)|^{2}\right\rangle$ obtained over a time period of $T=10^{4}$ for system size $L=78$ with $\epsilon=0.2$ is shown in Fig. 2. As can be seen, the dominant modes occur in the vicinity of $k= \pm 1$. Note, however, the smaller peak near zero wave number, which arises from coupling between unstable short-wavelength modes centered about $k= \pm 1$ and the slowly decaying modes from the Goldstone branch of the spectrum centered at $k=0$ (using terminology based upon the linear stability analysis of the spatially uniform solution). The power spectrum for $\hat{u}(k)=\hat{v}(k) / i k$ (shown in the inset in Fig. 2) has the

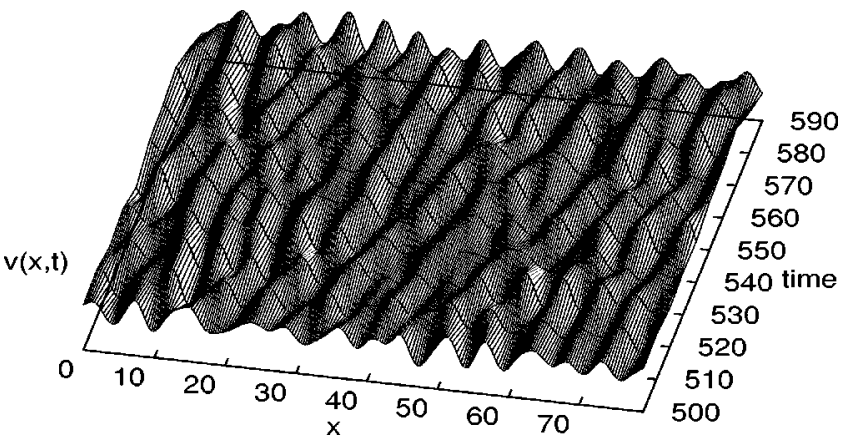

FIG. 1. Typical space-time configuration for $v(x, t)$ in the steady chaotic regime is shown here. The configuration shown has evolved from random initial condition within a system size $L=78$ and $\epsilon=0.5$.

dominant peak near $k=0$, as was originally demonstrated in [19]. One would also expect on general grounds [5] each Fourier transform variable $\hat{v}(k, \omega)$ to be governed by a Gaussian probability distribution functional, $\exp \left[-D\left(|\hat{v}(k, \omega)|^{2}\right)\right]$, for small $k$ and $\omega$. We have verified this for several values of $k$ and $\omega$ by fitting the probability distribution for the variable $z \equiv|\hat{v}(k)|^{2}$ to an exponential form: $f(z)=D \mathrm{e}^{-D z}$. For instance, in the particular case $L$ $=78, \epsilon=0.5$, we obtain a good fit for $\omega=0.6, k=1.5$ by using $D \approx 47$.

As has already been emphasized, the key question for any system exhibiting STC is whether it can be represented as a union of weakly correlated subsystems. If this is the case, then the spectrum of Lyapunov exponents for the entire system should be intensive in the sense that $\lambda_{i}$ is a function only of the intensive index $i / V$, i.e., $\lambda_{i}=f(i / V)$, where $V$ stands for the volume of the system [3]. The question is not trivial for the type of STC considered here, because of the importance of the long-wavelength modes and the divergence of the two point correlation length $\xi_{2}$ [33] as $\epsilon \rightarrow 0$ [20]. To answer this question, a detailed study of the Lyapunov spectrum for the Nikolaevskii model was conducted. The results are shown in Fig. 3 where the number of Lyapunov exponents greater than a particular value $\lambda_{i}$, scaled by the system size $L$, is plotted versus $\lambda_{i}$ for $\epsilon=0.5$. A similar curve is

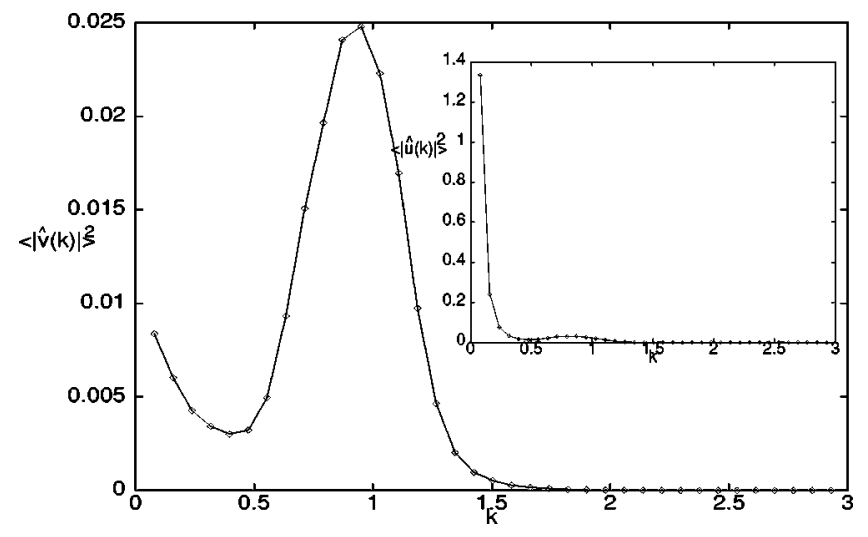

FIG. 2. Time average power spectra $\left\langle|\hat{v}(k)|^{2}\right\rangle$ and $\left\langle|\hat{u}(k)|^{2}\right\rangle$ in $k$-space are plotted. The system size is $L=78$ and control parameter $\epsilon=0.2$. Note that the power spectrum has symmetry with respect to $k \rightarrow-k$. 


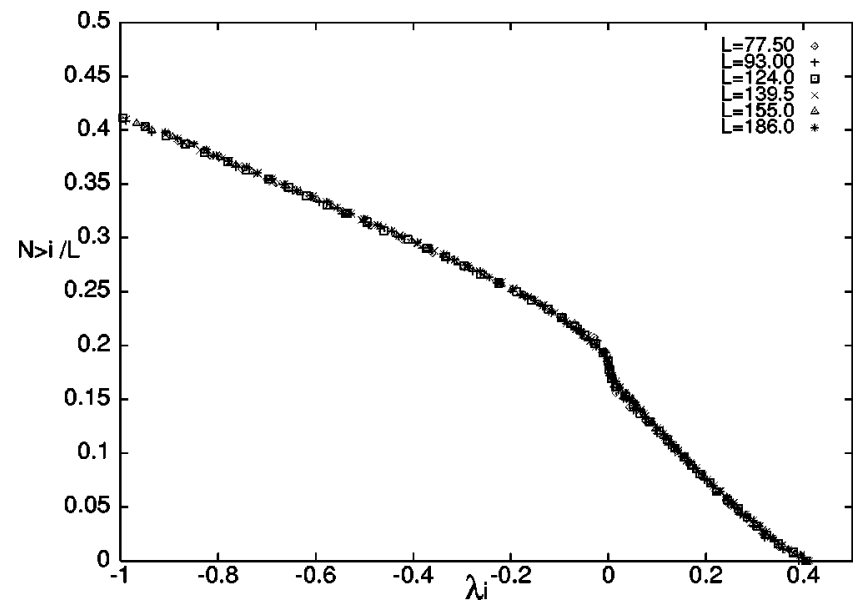

FIG. 3. Here $N_{>i}$ is the number of Lyapunov exponents greater than a particular value $\lambda_{i}$. We plot $N_{>i} / L$ (scaled by the system size $L$ ) vs $\lambda_{i}$ in the case $\epsilon=0.5$.

found at $\epsilon=0.2$, but the maximum positive eigenvalue is now smaller (one expects this eigenvalue to vanish as $\epsilon$ $\rightarrow 0$.) The intensive nature of the Lyapunov density is evident. In this case, one also expects the fractal dimension $D(L)$ of an attractor to be extensive for large enough $L$ (extensive chaos), as was first shown by Manneville [15] for chaotic solutions of the Kuramoto-Sivashinsky equation. We have checked this for the Nikolaevskii model using the Kaplan-Yorke formula [4] for the Lyapunov dimension,

$$
D(L)=K+\frac{1}{\left|\lambda_{K+1}\right|} \sum_{i=1}^{K} \lambda_{i},
$$

where the integer $K$ is the largest integer such that the sum of the first $K$ Lyapunov exponents is non-negative. We have also calculated the Kolmogorov-Sinai entropy $H(L)$ from the definition

$$
H(L)=\sum_{i=1}^{i_{+}} \lambda_{i},
$$

where the sum is over the positive Lyapunov exponents. The Kolmogorov-Sinai entropy [32] is a measure of the mean rate of information production in a system, or the mean rate of growth of uncertainty in a system subjected to small perturbations. We find that for large enough $L$ both $D(L)$ and $H(L)$ are extensive. Our results for the Lyapunov dimension $D(L)$ are shown in Fig. 4 for $\epsilon=0.5$. The same behavior is found at $\epsilon=0.2$, but with a different slope (naturally the slope of the straight line $D(L)$ at $\epsilon=0.2$ is smaller than that at $\epsilon=0.5)$. In addition, we find that the upper index $i_{+}$in Eq. (3), which corresponds to the smallest positive Lyapunov exponent, is also proportional to $L$.

The important characteristic of STC is the dimension correlation length $\xi_{\delta}[1,3]$. This length is defined as $\xi_{\delta} \equiv \delta^{-1 / d}$, where $\delta \equiv \lim _{L \rightarrow \infty} D(L) / L^{d}$. It can be thought of as the "radius" of a volume that contains one degree of freedom, or, as the linear size of the subsystem described above. The value of this dimension correlation length for Eq. (1) is $\xi_{\delta}$ $=3.0$ for $\epsilon=0.5$ and $\xi_{\delta}=3.3$ for $\epsilon=0.2$. In contrast to $\xi_{\delta}$ the

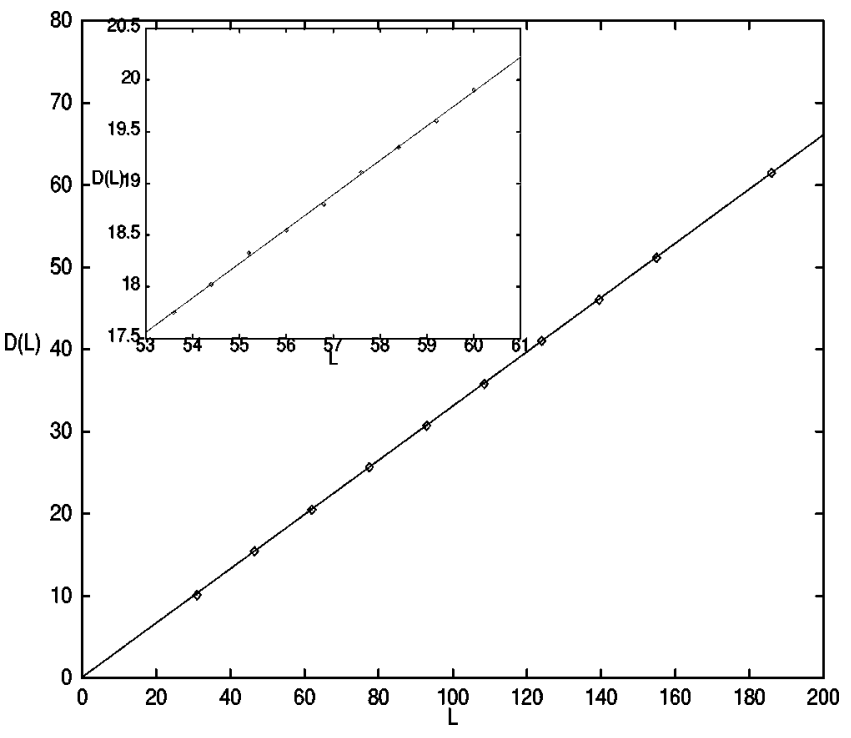

FIG. 4. Lyapunov dimension $D(L)$ vs system size $L$ for $\epsilon$ $=0.5$. $\delta L=15.5$ for extensive, and $\delta L=0.8$ for microextensive.

above-mentioned two point correlation length $\xi_{2}$ governs the spatial decay of the correlation function. In general these two lengths are different [3]. We found that $\xi_{2} \cong 4.9$ for the $\mathrm{Ni}$ kolaevskii model at $\epsilon=0.5$ and $\xi_{2} \cong 5.6$ at $\epsilon=0.2$.

The use of periodic boundary conditions ensures that the dynamics observed is not influenced by the boundaries and hence, in some sense, it is intrinsic to the system. Also, for a large enough system size, periodic boundary conditions approximate the thermodynamic limit of infinite size and have been used previously in many other similar studies $[34,16,15]$. It would be interesting to analyze the finite size effects introduced by the use of other types of boundary conditions, such as rigid-rigid. However, the fact that in all cases considered here the system size is much larger than any of the correlation lengths obtained, suggests to us that similar results would be obtained with other boundary conditions.

Finally, Tajima and Greenside [35] have recently found for the one dimensional Kuramoto-Sivashinsky model that $D(L)$ is also "microextensive." Namely, they found that if one increases $L$ by a small amount $\delta L$, with $\delta L \ll \xi_{\delta}(\delta L$ $=0.8$ in our simulation), one finds that $D(L)$ satisfies the same linear relationship as that characterizing extensive chaos. We have examined this for two different domains of $L$ for our model and found that microextensivity holds for both $D(L)$ and $H(L)$. Our results for microextensivity for $D(L)$ are shown in the inset in Fig. 4.

In conclusion, our detailed study of this type of STC based on the numerical integration of the Nikolaevskii model shows that for sufficiently large system size the chaos is both extensive and microextensive. We also found that the system satisfies Gaussian statistics at sufficiently small wave numbers and frequencies. We believe these results are quite general and reflect intrinsic features of this type of STC, rather than specific peculiarities of the model.

There are several interesting questions to investigate in the limit $\epsilon \rightarrow 0$, including the dependence of quantities such as the correlation lengths and Lyapunov exponents on $\epsilon$ as 
well as the possible scaling of the power spectrum. This study is in progress and will be reported elsewhere.

We would like to thank Henry Greenside for a helpful discussion. This work was supported by NATO Grant No. CRG.CRG972822, by NSF Grant No. DMR9810409, Project Nos. PB94-1167 and PB97-0141-C02-01 (Spain), and the
Grant-in-Aid for Scientific Research (No. 11837006) from the Ministry of Education, Science, Sports and Culture (Japan). We also wish to acknowledge an allocation of time on the Pittsburgh Supercomputer Center, where some of this work was carried out. J.D.G. acknowledges support from the Fundación Banco Bilbao Vizcaya in the Programa Cátedra.
[1] M. C. Cross and P. C. Hohenberg, Rev. Mod. Phys. 65, 851 (1993).

[2] P. Manneville, Dissipative Structures and Weak Turbulence (Academic Press, New York, 1990).

[3] H. Greenside, in Proceedings of the CRM Workshop, SemiAnalytic Methods for the Navier Stokes Equations, edited by K. Coughlin (American Mathematical Society, Providence, RI, 1999), and references therein.

[4] J. L. Kaplan and J. A. Yorke, Chaotic Behavior of Multidimensional Difference Equations, Lecture Notes in Mathematics Vol. 730 (Springer, New York, 1979).

[5] P. C. Hohenberg and B. I. Shraiman, Physica D 37, 109 (1989).

[6] D. Ruelle, Commun. Math Phys. 87, 287 (1982).

[7] V. Yakhot, Phys. Rev. A 24, 642 (1989).

[8] V. S. L'vov, V. V. Lebedev, M. Paton, and I. Procaccia, Nonlinearity 6, 25 (1993).

[9] C. C. Chow and T. Hwa, Physica D 84, 494 (1995).

[10] R. W. Wittenberg and P. Holmes, Chaos 9, 452 (199).

[11] R. Carretero-Gonzalez, S. Orstavik, J. Huke, D. S. Broomhead, and J. Stark, Chaos 9, 466 (1999).

[12] R. Livi, A. Politi, and S. Ruffo, J. Phys. A 25, 4813 (1992).

[13] H. Chate, Europhys. Lett. 21, 419 (1993).

[14] A. Pikovsky and A. Politi, Nonlinearity 11, 1049 (1998).

[15] P. Manneville, in Macroscopic Modeling of Turbulent Flows, edited by O. Pironneau, Lecture Notes in Physics Vol. 230 (Springer-Verlag, Berlin, 1985), p. 319.

[16] D. A. Egolf and H. S. Greenside, Phys. Rev. Lett. 74, 1751 (1995).

[17] H. Richter, N. Kloepper, A. Hertrich, and A. Buka, Europhys. Lett. 30, 37 (1995).

[18] S. Kai, K. Hayashi, and Y. Hidaka, J. Phys. Chem. 100, 19007 (1996).

[19] M. I. Tribelsky and K. Tsuboi, Phys. Rev. Lett. 76, 1631 (1996).

[20] M. I. Tribelskii, Usp. Fiz. Nauk. 167, 167 (1997) [Phys. Usp. 40, 159 (1997)].

[21] E. D. Siggia and A. Zippelius, Phys. Rev. Lett. 47, 835 (1981).

[22] F. H. Busse and E. W. Bolton, J. Fluid Mech. 146, 115 (1984).

[23] H. W. Xi, X. J. Li, and J. D. Gunton, Phys. Rev. Lett. 78, 1046 (1997).
[24] It is relevant to mention that the consideration of convection with the stress-free boundary conditions [21,22] was the very first analysis of pattern formation in the degenerate systems. However, at that time the dramatic destabilization of steady patterns obtained in this case was regarded as a peculiarity of the particular problem, rather than a generic attribute of the degeneracy.

[25] V. N. Nikolaevskii, in Recent Advances in Engineering Science, edited by S. L. Koh and C. G. Speciale, Lecture Notes in Engineering Vol. 39 (Springer-Verlag, New York, 1989), p. 210.

[26] I. A. Beresnev and V. N. Nikolaevskii, Physica D 66, 1 (1993).

[27] B. A. Malomed, Phys. Rev. A 45, 1009 (1992).

[28] M. I. Tribelsky and M. G. Velarde, Phys. Rev. E 54, 4973 (1996).

[29] M. I. Tribelsky, Int. J. Bifurcation Chaos Appl. Sci. Eng. 7, 997 (1997).

[30] I. L. Kliakhandler and B. A. Malomed, Phys. Lett. A 231, 191 (1997).

[31] Although the original numerical study [19] was carried out in terms of the order parameter $u(x, t)$, we have found that Eq. (1) is more convenient for numerical study as it exhibits greater numerical stability. The reason is that the conservation law $d \bar{v} / d t=0$ holding for this equation (where the bar denotes a spatial average) helps to stabilize the code. In our simulations the value $\bar{v}=0$ was imposed by the initial, random, conditions. For the space discretization we have found that $\Delta x$ $=0.31$ provides a good enough resolution.

[32] T. S. Parker and L. O. Chua, Practical Numerical Algorithms for Chaotic Systems (Springer-Verlag, New York, 1989).

[33] At small $\epsilon$ in this model the two point correlation function $\left(\left\langle v(x, t) v\left(x+x^{\prime}, t\right)\right\rangle\right.$, where the $\langle\ldots\rangle$ denotes the time average) is generally oscillatory with a spatial period of the oscillations close to $2 \pi$ and a slow decay of its amplitude over a scale $\xi_{2}$. Thus, the model has two correlation length scales, with $\xi_{2}$ being the larger of the two.

[34] B. I. Shraiman, A. Pumir, W. van Saarlos, P. C. Hohenberg, H. Chaté, and M. Holen, Physica D 57, 241 (1992).

[35] S. Tajima and H. S. Greenside, Bull. Am. Phys. Soc. 44(1), 327 (1999). 